\title{
Generation of Finite Difference Formulas on Arbitrarily Spaced Grids
}

\author{
By Bengt Fornberg
}

\begin{abstract}
Simple recursions are derived for calculating the weights in compact finite difference formulas for any order of derivative and to any order of accuracy on onedimensional grids with arbitrary spacing. Tables are included for some special cases (of equispaced grids).
\end{abstract}

1. Introduction. Previously published methods to generate finite difference weights (e.g., references [1]-[5]) have been of considerable complexity and often been limited to derivatives of low order on equidistantly spaced grids. The most ambitious attempt to tabulate weights for many orders of derivatives and to high orders of accuracy appears to be the work by Keller and Pereyra [4]. However, their algorithms (limited to equispaced grids) were very involved, and the resulting tables contain both isolated and systematic errors.

In the present study we describe two simple recursion relations which give the weights for any order of derivative (including the 0th derivative, corresponding to interpolation), approximated to any order of accuracy on an arbitrary grid in one dimension. Since, in general, only four arithmetic operations are needed to determine each weight, the main anticipated application of the present method is to dynamically changing grids. However, the method is also well suited to generate tables of weights. Such tables (in the special case of equispaced grids, up to the 4th derivative and up to 9 weights) are included in the cases of one-sided and centered approximations at a grid point and at a 'half-way point' between grid points.

2. Notation, Algorithm. Given $M \geq 0$, the order of the highest derivative we wish to approximate, and a set of $N+1$ grid points (at $x$-coordinates $\alpha_{0}, \ldots, \alpha_{N}$; $N \geq 0$ ), the problem is to find all the weights such that the approximations

$$
\left.\frac{d^{m} f}{d x^{m}}\right|_{x=x_{0}} \approx \sum_{\nu=0}^{n} \delta_{n, \nu}^{m} f\left(\alpha_{\nu}\right), \quad m=0,1, \ldots, M ; n=m, m+1, \ldots, N
$$

become of optimal formal order of accuracy (in general of order $n-m+1$, although it can be higher in special cases). The following algorithm achieves this:

Received May 20, 1987; revised December 28, 1987.

1980 Mathematics Subject (Classification (1985 Revision). Primary 65D25.

Key words and phrases. Finite difference coefficients, high-order accuracy. 


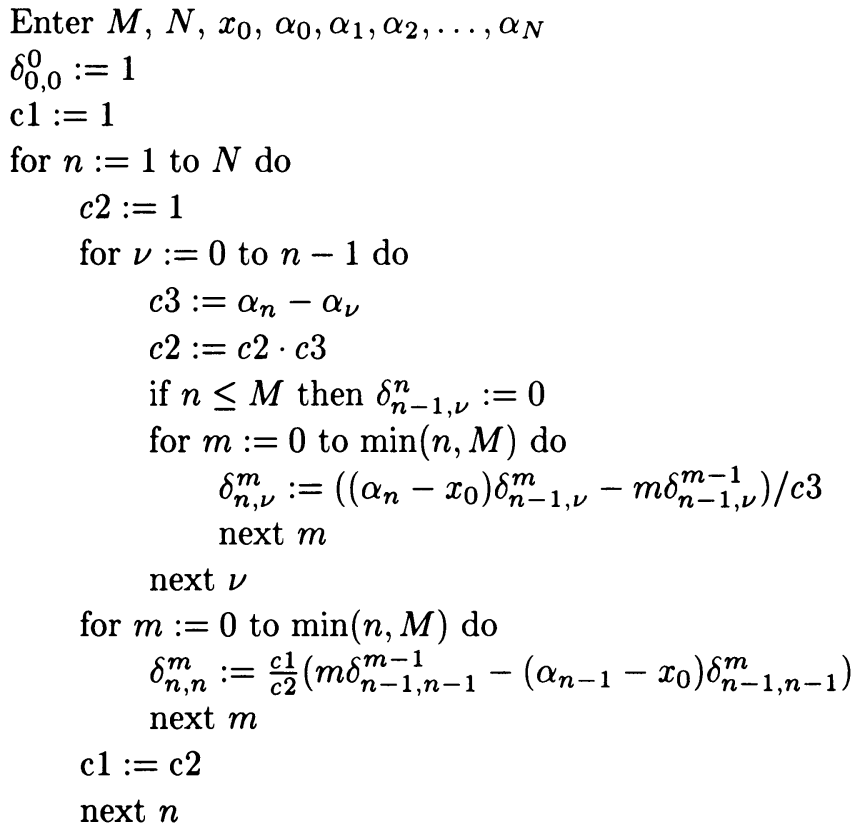

Notes. 1. If the array $\delta_{n, \nu}^{m}$ initially is zero, the statement "if $n \leq M$ then $\delta_{n-1, \nu}^{n}:=0$ " can be omitted.

2. In the case of $m=0$ (corresponding to interpolation formulas), expressions of the form 'zero*(undefined number)' occur. The result is assumed to be zero.

3. The order in which the $\alpha_{\nu}$ (all distinct) are given is significant (since the weights corresponding to all leading subsets of the $\alpha_{\nu}$ 's are calculated). There is no restriction on $x_{0}$ coinciding with any $\alpha_{\nu}$.

3. Derivation of the Algorithm. For simplicity, assume we seek to approximate the derivatives at the point $x_{0}=0$. Let $\left\{\alpha_{0}, \alpha_{1}, \ldots, \alpha_{N}\right\}$ be distinct real numbers and denote

$$
\omega_{n}(x):=\prod_{k=0}^{n}\left(x-\alpha_{k}\right)
$$

The polynomial

$$
F_{n, \nu}(x):=\frac{\omega_{n}(x)}{\omega_{n}^{\prime}\left(\alpha_{\nu}\right)\left(x-\alpha_{\nu}\right)}
$$

is the one of minimal degree which takes the value 1 at $x=\alpha_{\nu}$ and 0 at $x=\alpha_{k}$, $0 \leq k \leq n, k \neq \nu$. For an arbitrary function $f(x)$ and nodes $x=\alpha_{\nu}$, Lagrange's interpolation polynomial becomes

$$
p(x):=\sum_{\nu=0}^{n} F_{n, \nu}(x) f\left(\alpha_{\nu}\right) .
$$

The desired weights express how the values of $\left[d^{m} p(x) / d x^{m}\right]_{x=0}$ vary with changes in $f\left(\alpha_{\nu}\right)$. Since only one term in $p(x)$ is influenced by changes in each $f\left(\alpha_{\nu}\right)$, we find

$$
\delta_{n, \nu}^{m}=\left[\frac{d^{m}}{d x^{m}} F_{n, \nu}(x)\right]_{x=0}
$$


Therefore, the $n$th degree polynomial $F_{n, \nu}(x)$ can also be expressed as

$$
F_{n, \nu}(x)=\sum_{m=0}^{n} \frac{\delta_{n, \nu}^{m}}{m !} x^{m}
$$

From (3.2) follow (noting that $\omega(x)=\left(x-\alpha_{n}\right) \omega_{n-1}(x)$ implies $\omega_{n}^{\prime}(x)=(x-$ $\left.\left.\alpha_{n}\right) \omega_{n-1}^{\prime}(x)+\omega_{n-1}(x)\right)$

$$
F_{n, \nu}(x)=\frac{x-\alpha_{n}}{\alpha_{\nu}-\alpha_{n}} F_{n-1, \nu}(x)
$$

and

$$
F_{n, n}(x)=\frac{\omega_{n-1}(x)}{\omega_{n-1}\left(\alpha_{n}\right)}=\frac{\omega_{n-2}\left(\alpha_{n-1}\right)}{\omega_{n-1}\left(\alpha_{n}\right)}\left(x-\alpha_{n-1}\right) F_{n-1, n-1}(x) \quad(n>1) .
$$

By substituting the expansion (3.5) into (3.6) and (3.7), and by equating powers of $x$, the desired recursion relations between the weights are obtained:

$$
\delta_{n, \nu}^{m}=\frac{1}{\alpha_{n}-\alpha_{\nu}}\left(\alpha_{n} \delta_{n-1, \nu}^{m}-m \delta_{n-1, \nu}^{m-1}\right)
$$

and

$$
\delta_{n, n}^{m}=\frac{\omega_{n-2}\left(\alpha_{n-1}\right)}{\omega_{n-1}\left(\alpha_{n}\right)}\left(m \delta_{n-1, n-1}^{m-1}-\alpha_{n-1} \delta_{n-1, n-1}^{m}\right) .
$$

The relation

$$
\sum_{\nu=0}^{n} \delta_{n, \nu}^{m}= \begin{cases}1, & m=0 \\ 0, & m>0\end{cases}
$$

could be used instead of (3.9) to obtain $\delta_{n, n}^{m}$. However, this would increase the operation count and might also cause a growth of errors in the case of floatingpoint arithmetic.

4. Description of the Tables. Special cases which commonly occur are centered and one-sided approximations on equidistant grids. The particular choices of $\alpha_{\nu}$ used for Tables 1-4 correspond to grid spacings $\Delta x=1$. For other values of $\Delta x$, these coefficients should be divided by $(\Delta x)^{m}$ (where $m$, as before, is the order of the derivative). 
TABLE 1

Some weights for centered approximations at a grid point (generated by setting $M=$ $4, N=8, x_{0}=0$ and $\left.\alpha_{\nu}=\{0,1,-1,2,-2,3,-3,4,-4\}\right)$.

\begin{tabular}{|c|c|c|c|c|c|c|c|c|c|c|}
\hline 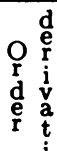 & $\begin{array}{lll}0 & & \\
r & a \\
d & a \\
d & c \\
\mathbf{e} & c \\
r & \mathbf{r}\end{array}$ & & $\begin{array}{l}\text { pprox } \\
\text {-coord }\end{array}$ & $\begin{array}{l}\text { lations } \\
\text { ates at }\end{array}$ & $\begin{array}{c}x=0 \\
\text { odes: }\end{array}$ & & & & & \\
\hline $\begin{array}{l}0 \\
\text { f } \\
\text { ve }\end{array}$ & $\mid \begin{array}{lll}0 & c \\
f & c\end{array}$ & -4 & -3 & -2 & -1 & 0 & 1 & 2 & 3 & 4 \\
\hline 0 & $\infty$ & & & & & 1 & & & & \\
\hline & 2 & & & & $\frac{-1}{2}$ & 0 & $\frac{1}{2}$ & & & \\
\hline & 4 & & & $\frac{1}{12}$ & $\frac{-2}{3}$ & 0 & $\frac{2}{3}$ & $\frac{-1}{12}$ & & \\
\hline 1 & 6 & & $\frac{-1}{60}$ & $\frac{3}{20}$ & $\frac{-3}{4}$ & 0 & $\frac{3}{4}$ & $\frac{-3}{20}$ & $\frac{1}{60}$ & \\
\hline & 8 & $\frac{1}{280}$ & $\frac{-4}{105}$ & $\frac{1}{5}$ & $\frac{-4}{5}$ & 0 & $\frac{4}{5}$ & $\frac{-1}{5}$ & $\frac{4}{105}$ & $\frac{-1}{280}$ \\
\hline & 2 & & & & 1 & -2 & 1 & & & \\
\hline & 4 & & & $\frac{-1}{12}$ & $\frac{4}{3}$ & $\frac{-5}{2}$ & $\frac{4}{3}$ & $\frac{-1}{12}$ & & \\
\hline 2 & 6 & & $\frac{1}{90}$ & $\frac{-3}{20}$ & $\frac{3}{2}$ & $\frac{-49}{18}$ & $\frac{3}{2}$ & $\frac{-3}{20}$ & $\frac{1}{90}$ & \\
\hline & 8 & $\frac{-1}{560}$ & $\frac{8}{315}$ & $\frac{-1}{5}$ & $\frac{8}{5}$ & $\frac{-205}{72}$ & $\frac{8}{5}$ & $\frac{-1}{5}$ & $\frac{8}{315}$ & $\frac{-1}{560}$ \\
\hline & 2 & & & $\frac{-1}{2}$ & 1 & 0 & -1 & $\frac{1}{2}$ & & \\
\hline 3 & 4 & & $\frac{1}{8}$ & -1 & $\frac{13}{8}$ & 0 & $\frac{-13}{8}$ & 1 & $\frac{-1}{8}$ & \\
\hline & 6 & $\frac{-7}{240}$ & $\frac{3}{10}$ & $\frac{-169}{120}$ & $\frac{61}{30}$ & 0 & $\frac{-61}{30}$ & $\frac{169}{120}$ & $\frac{-3}{10}$ & $\frac{7}{240}$ \\
\hline & 2 & & & 1 & -4 & 6 & -4 & 1 & & \\
\hline 4 & 4 & & $\frac{-1}{6}$ & 2 & $\frac{-13}{2}$ & $\frac{28}{3}$ & $\frac{-13}{2}$ & 2 & $\frac{-1}{6}$ & \\
\hline & 6 & $\frac{7}{240}$ & $\frac{-2}{5}$ & $\frac{169}{60}$ & $\frac{-122}{15}$ & $\frac{91}{8}$ & $\frac{-122}{15}$ & $\frac{169}{60}$ & $\frac{-2}{5}$ & $\frac{7}{240}$ \\
\hline
\end{tabular}


TABLE 2

Some weights for centered approximations at a 'half-way' point (generated by setting $M=4, N=7, x_{0}=0$ and $\left.\alpha_{\nu}=\{1 / 2,-1 / 2,3 / 2,-3 / 2,5 / 2,-5 / 2,7 / 2,-7 / 2\}\right)$.

\begin{tabular}{|c|c|c|c|c|c|c|c|c|c|}
\hline 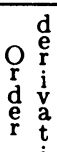 & $\begin{array}{ll}O & \\
\mathrm{r} & \mathrm{a} \\
\mathrm{d} & \mathrm{c} \\
\mathrm{e} & \mathrm{c} \\
\mathrm{r} & \mathrm{u} \\
\mathrm{r}\end{array}$ & & $\begin{array}{l}\text { Approx } \\
x \text {-coord }\end{array}$ & $\begin{array}{l}\text { mations } \\
\text { nates at }\end{array}$ & $\begin{array}{l}x=0 \\
\text { todes: }\end{array}$ & & & & \\
\hline $\begin{array}{l}0 \mathrm{v} \\
\mathrm{f} \\
\mathrm{v}\end{array}$ & $\begin{array}{ll}0 & c \\
f & c \\
y\end{array}$ & $-7 / 2$ & $-5 / 2$ & $-3 / 2$ & $-1 / 2$ & $1 / 2$ & $3 / 2$ & $5 / 2$ & $7 / 2$ \\
\hline & 2 & & & & $\frac{1}{2}$ & $\frac{1}{2}$ & & & \\
\hline & 4 & & & $\frac{-1}{16}$ & $\frac{9}{16}$ & $\frac{9}{16}$ & $\frac{-1}{16}$ & & \\
\hline 0 & 6 & & $\frac{3}{256}$ & $\frac{-25}{256}$ & $\frac{75}{128}$ & $\frac{75}{128}$ & $\frac{-25}{256}$ & $\frac{3}{256}$ & \\
\hline & 8 & $\frac{-5}{2048}$ & $\frac{49}{2048}$ & $\frac{-245}{2048}$ & $\frac{1225}{2048}$ & $\frac{1225}{2048}$ & $\frac{-245}{2048}$ & $\frac{49}{2048}$ & $\frac{-5}{2048}$ \\
\hline & 2 & & & & -1 & 1 & & & \\
\hline & 4 & & & $\frac{1}{24}$ & $\frac{-9}{8}$ & $\frac{9}{8}$ & $\frac{-1}{24}$ & & \\
\hline 1 & 6 & & $\frac{-3}{640}$ & $\frac{25}{384}$ & $\frac{-75}{64}$ & $\frac{75}{64}$ & $\frac{-25}{384}$ & $\frac{3}{640}$ & \\
\hline & 8 & $\frac{5}{7168}$ & $\frac{-49}{5120}$ & $\frac{245}{3072}$ & $\frac{-1225}{1024}$ & $\frac{1225}{1024}$ & $\frac{-245}{3072}$ & $\frac{49}{5120}$ & $\frac{-5}{7168}$ \\
\hline & 2 & & & $\frac{1}{2}$ & $\frac{-1}{2}$ & $\frac{-1}{2}$ & $\frac{1}{2}$ & & \\
\hline 2 & 4 & & $\frac{-5}{48}$ & $\frac{13}{16}$ & $\frac{-17}{24}$ & $\frac{-17}{24}$ & $\frac{13}{16}$ & $\frac{-5}{48}$ & \\
\hline & 6 & $\frac{259}{11520}$ & $\frac{-499}{2304}$ & $\frac{1299}{1280}$ & $\frac{-1891}{2304}$ & $\frac{-1891}{2304}$ & $\frac{1299}{1280}$ & $\frac{-499}{2304}$ & $\frac{259}{11520}$ \\
\hline & 2 & & & -1 & 3 & -3 & 1 & & \\
\hline 3 & 4 & & $\frac{1}{8}$ & $\frac{-13}{8}$ & $\frac{17}{4}$ & $\frac{-17}{4}$ & $\frac{13}{8}$ & $\frac{-1}{8}$ & \\
\hline & 6 & $\frac{-37}{1920}$ & $\frac{499}{1920}$ & $\frac{-1299}{640}$ & $\frac{1891}{384}$ & $\frac{-1891}{384}$ & $\frac{1299}{640}$ & $\frac{-499}{1920}$ & $\frac{37}{1920}$ \\
\hline & 2 & & $\frac{1}{2}$ & $\frac{-3}{2}$ & 1 & 1 & $\frac{-3}{2}$ & $\frac{1}{2}$ & \\
\hline 4 & 4 & $\frac{-7}{48}$ & $\frac{59}{48}$ & $\frac{-45}{16}$ & $\frac{83}{48}$ & $\frac{83}{48}$ & $\frac{-45}{16}$ & $\frac{59}{48}$ & $\frac{-7}{48}$ \\
\hline
\end{tabular}


TABLE 3

Some weights for one-sided approximations at a grid point (generated by setting $M=4, N=8, x_{0}=0$ and $\left.\alpha_{\nu}=\{0,1,2,3,4,5,6,7,8\}\right)$.

\begin{tabular}{|c|c|c|c|c|c|c|c|c|c|c|}
\hline \multirow{2}{*}{ 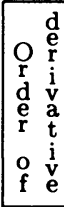 } & \multirow{2}{*}{$\begin{array}{ll}O \\
\mathrm{r} \\
\mathrm{r} \\
\mathrm{d} & \mathrm{c} \\
\mathrm{e} & \mathrm{c} \\
\mathrm{r} & \mathrm{u} \\
\mathrm{r} & \mathrm{r} \\
\mathrm{o} & \mathrm{c} \\
\mathrm{f} & \mathrm{y}\end{array}$} & \multicolumn{9}{|c|}{$\begin{array}{l}\text { Approximations at } x=0 \\
x \text {-coordinates at nodes: }\end{array}$} \\
\hline & & 0 & 1 & 2 & 3 & 4 & 5 & 6 & 7 & 8 \\
\hline 0 & $\infty$ & 1 & & & & & & & & \\
\hline \multirow{8}{*}{1} & 1 & -1 & 1 & & & & & & & \\
\hline & 2 & $\frac{-3}{2}$ & 2 & $\frac{-1}{2}$ & & & & & & \\
\hline & 3 & $\frac{-11}{6}$ & 3 & $\frac{-3}{2}$ & $\frac{1}{3}$ & & & & & \\
\hline & 4 & $\frac{-25}{12}$ & 4 & -3 & $\frac{4}{3}$ & $\frac{-1}{4}$ & & & & \\
\hline & 5 & $\frac{-137}{60}$ & 5 & -5 & $\frac{10}{3}$ & $\frac{-5}{4}$ & $\frac{1}{5}$ & & & \\
\hline & 6 & $\frac{-49}{20}$ & 6 & $\frac{-15}{2}$ & $\frac{20}{3}$ & $\frac{-15}{4}$ & $\frac{6}{5}$ & $\frac{-1}{6}$ & & \\
\hline & 7 & $\frac{-363}{140}$ & 7 & $\frac{-21}{2}$ & $\frac{35}{3}$ & $\frac{-35}{4}$ & $\frac{21}{5}$ & $\frac{-7}{6}$ & $\frac{1}{7}$ & \\
\hline & 8 & $\frac{-761}{280}$ & 8 & -14 & $\frac{56}{3}$ & $\frac{-35}{2}$ & $\frac{56}{5}$ & $\frac{-14}{3}$ & $\frac{8}{7}$ & $\frac{-1}{8}$ \\
\hline \multirow{7}{*}{2} & 1 & 1 & -2 & 1 & & & & & & \\
\hline & 2 & 2 & -5 & 4 & -1 & & & & & \\
\hline & 3 & $\frac{35}{12}$ & $\frac{-26}{3}$ & $\frac{19}{2}$ & $\frac{-14}{3}$ & $\frac{11}{12}$ & & & & \\
\hline & 4 & $\frac{15}{4}$ & $\frac{-77}{6}$ & $\frac{107}{6}$ & -13 & $\frac{61}{12}$ & $\frac{-5}{6}$ & & & \\
\hline & 5 & $\frac{203}{45}$ & $\frac{-87}{5}$ & $\frac{117}{4}$ & $\frac{-254}{9}$ & $\frac{33}{2}$ & $\frac{-27}{5}$ & $\frac{137}{180}$ & & \\
\hline & 6 & $\frac{469}{90}$ & $\frac{-223}{10}$ & $\frac{879}{20}$ & $\frac{-949}{18}$ & 41 & $\frac{-201}{10}$ & $\frac{1019}{180}$ & $\frac{-7}{10}$ & \\
\hline & 7 & $\frac{29531}{5040}$ & $\frac{-962}{35}$ & $\frac{621}{10}$ & $\frac{-4006}{45}$ & $\frac{691}{8}$ & $\frac{-282}{5}$ & $\frac{2143}{90}$ & $\frac{-206}{35}$ & $\frac{363}{560}$ \\
\hline \multirow{6}{*}{3} & 1 & -1 & 3 & -3 & 1 & & & & & \\
\hline & 2 & $\frac{-5}{2}$ & 9 & -12 & 7 & $\frac{-3}{2}$ & & & & \\
\hline & 3 & $\frac{-17}{4}$ & $\frac{71}{4}$ & $\frac{-59}{2}$ & $\frac{49}{2}$ & $\frac{-41}{4}$ & $\frac{7}{4}$ & & & \\
\hline & 4 & $\frac{-49}{8}$ & 29 & $\frac{-461}{8}$ & 62 & $\frac{-307}{8}$ & 13 & $\frac{-15}{8}$ & & \\
\hline & 5 & $\frac{-967}{120}$ & $\frac{638}{15}$ & $\frac{-3929}{40}$ & $\frac{389}{3}$ & $\frac{-2545}{24}$ & $\frac{268}{5}$ & $\frac{-1849}{120}$ & $\frac{29}{15}$ & \\
\hline & 6 & $\frac{-801}{80}$ & $\frac{349}{6}$ & $\frac{-18353}{120}$ & $\frac{2391}{10}$ & $\frac{-1457}{6}$ & $\frac{4891}{30}$ & $\frac{-561}{8}$ & $\frac{527}{30}$ & $\frac{-469}{240}$ \\
\hline \multirow{5}{*}{4} & 1 & 1 & -4 & 6 & -4 & 1 & & & & \\
\hline & 2 & 3 & -14 & 26 & -24 & 11 & -2 & & & \\
\hline & 3 & $\frac{35}{6}$ & -31 & $\frac{137}{2}$ & $\frac{-242}{3}$ & $\frac{107}{2}$ & -19 & $\frac{17}{6}$ & & \\
\hline & 4 & $\frac{28}{3}$ & $\frac{-111}{2}$ & 142 & $\frac{-1219}{6}$ & 176 & $\frac{-185}{2}$ & $\frac{82}{3}$ & $\frac{-7}{2}$ & \\
\hline & 5 & $\frac{1069}{80}$ & $\frac{-1316}{15}$ & $\frac{15289}{60}$ & $\frac{-2144}{5}$ & $\frac{10993}{24}$ & $\frac{-4772}{15}$ & $\frac{2803}{20}$ & $\frac{-536}{15}$ & $\frac{967}{240}$ \\
\hline
\end{tabular}


TABLE 4

Some weights for one-sided approximations at a 'half-way' point (generated by setting $M=4, N=8, x_{0}=0$ and $\left.\alpha_{\nu}=\{-1 / 2,1 / 2,3 / 2,5 / 2,7 / 2,9 / 2,11 / 2,13 / 2,15 / 2\}\right)$.

\begin{tabular}{|c|c|c|c|c|c|c|c|c|c|c|}
\hline \multirow{2}{*}{ 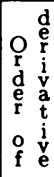 } & \multirow{2}{*}{ 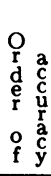 } & \multicolumn{9}{|c|}{$\begin{array}{l}\text { Approximations at } x=0 \\
x \text {-coordinates at nodes: }\end{array}$} \\
\hline & & $-1 / 2$ & $1 / 2$ & $3 / 2$ & $5 / 2$ & $7 / 2$ & $9 / 2$ & $11 / 2$ & $13 / 2$ & $15 / 2$ \\
\hline \multirow{10}{*}{0} & 1 & 1 & & & & & & & & \\
\hline & 2 & $\frac{1}{2}$ & $\frac{1}{2}$ & & & & & & & \\
\hline & 3 & $\frac{3}{8}$ & $\frac{3}{4}$ & $\frac{-1}{8}$ & & & & & & \\
\hline & 4 & $\frac{5}{16}$ & $\frac{15}{16}$ & $\frac{-5}{16}$ & $\frac{1}{16}$ & & & & & \\
\hline & 5 & $\frac{35}{128}$ & $\frac{35}{32}$ & $\frac{-35}{64}$ & $\frac{7}{32}$ & $\frac{-5}{128}$ & & & & \\
\hline & 6 & $\frac{63}{256}$ & $\frac{315}{256}$ & $\frac{-105}{128}$ & $\frac{63}{128}$ & $\frac{-45}{256}$ & $\frac{7}{256}$ & & & \\
\hline & 7 & $\frac{231}{1024}$ & $\frac{693}{512}$ & $\frac{-1155}{104}$ & $\frac{231}{256}$ & $\frac{-495}{1024}$ & $\frac{77}{512}$ & $\frac{-21}{1024}$ & & \\
\hline & 8 & $\frac{429}{3010}$ & $\frac{3003}{2010}$ & $\begin{array}{r}10003 \\
-3003 \\
2010\end{array}$ & $\begin{array}{r}2000 \\
3003 \\
2019\end{array}$ & $\begin{array}{r}1024 \\
-2145 \\
019\end{array}$ & $\frac{1001}{2019}$ & $\frac{-273}{0.020}$ & $\frac{33}{20}$ & \\
\hline & & $\begin{array}{r}2048 \\
6435 \\
\end{array}$ & $\begin{array}{l}2048 \\
6435 \\
\end{array}$ & $\begin{array}{r}2048 \\
-15015 \\
\end{array}$ & $\begin{array}{l}2048 \\
9009 \\
\end{array}$ & $\begin{array}{r}2048 \\
-32175 \\
\end{array}$ & $\begin{array}{r}2048 \\
5005 \\
\end{array}$ & $\begin{array}{r}2048 \\
-4095 \\
\end{array}$ & $\begin{array}{r}2048 \\
495 \\
\end{array}$ & -429 \\
\hline & 9 & $\frac{6435}{32768}$ & $\frac{6435}{4096}$ & $\frac{-10010}{8192}$ & $\begin{array}{l}\frac{9009}{4096} \\
\end{array}$ & $\frac{-52110}{16384}$ & $\frac{5000}{4096}$ & $\begin{array}{r}\frac{-4095}{8192} \\
\end{array}$ & $\frac{450}{4096}$ & $\frac{-429}{32768}$ \\
\hline \multirow{8}{*}{1} & 2 & -1 & 1 & & & & & & & \\
\hline & 3 & $\frac{-23}{24}$ & $\frac{7}{8}$ & $\frac{1}{8}$ & $\frac{-1}{24}$ & & & & & \\
\hline & 4 & $\frac{-11}{12}$ & $\frac{17}{24}$ & $\frac{3}{8}$ & $\frac{-5}{24}$ & $\frac{1}{24}$ & & & & \\
\hline & 5 & $\frac{-563}{640}$ & $\frac{67}{128}$ & $\frac{143}{192}$ & $\frac{-37}{64}$ & $\frac{29}{128}$ & $\frac{-71}{1920}$ & & & \\
\hline & 6 & $\frac{-1627}{1920}$ & $\frac{211}{640}$ & $\frac{59}{48}$ & $\frac{-235}{102}$ & $\frac{91}{108}$ & $\frac{-443}{1020}$ & $\frac{31}{0.60}$ & & \\
\hline & 7 & -88069 & 2021 & 28009 & -6803 & 5227 & $\begin{array}{r}-12673 \\
\end{array}$ & $\begin{array}{r}3539 \\
\end{array}$ & -3043 & \\
\hline & 7 & $\overline{107520}$ & 15360 & $\overline{15360}$ & $\overline{3072}$ & 3072 & 15360 & $\frac{2000}{15360}$ & $\overline{107520}$ & \\
\hline & 8 & $\frac{-1423}{1792}$ & $\frac{-491}{7168}$ & $\frac{7753}{3072}$ & $\frac{-18509}{5120}$ & $\frac{3535}{1024}$ & $\frac{-2279}{1024}$ & $\frac{953}{1024}$ & $\frac{-1637}{7168}$ & $\frac{2689}{107520}$ \\
\hline \multirow{8}{*}{2} & 1 & 1 & -2 & 1 & & & & & & \\
\hline & 2 & $\frac{3}{2}$ & $\frac{-7}{2}$ & $\frac{5}{2}$ & $\frac{-1}{2}$ & & & & & \\
\hline & 3 & $\frac{43}{24}$ & $\frac{-14}{3}$ & $\frac{17}{4}$ & $\frac{-5}{3}$ & $\frac{7}{24}$ & & & & \\
\hline & 4 & $\frac{95}{48}$ & $\frac{-269}{48}$ & $\frac{49}{8}$ & $\frac{-85}{24}$ & $\frac{59}{48}$ & $\frac{-3}{16}$ & & & \\
\hline & 5 & $\frac{12139}{5760}$ & $\begin{array}{r}-6119 \\
090\end{array}$ & $\frac{3091}{204}$ & $\begin{array}{r}-1759 \\
-209 \\
09\end{array}$ & $\frac{1211}{390}$ & $\begin{array}{r}-919 \\
060\end{array}$ & $\frac{739}{5790}$ & & \\
\hline & C & 25333 & $\begin{array}{r}-80813 \\
\end{array}$ & $\begin{array}{r}384 \\
2553\end{array}$ & $\begin{array}{r}288 \\
-21457\end{array}$ & $\begin{array}{r}384 \\
14651\end{array}$ & $\begin{array}{r}960 \\
-3687\end{array}$ & $\begin{array}{l}5760 \\
8863\end{array}$ & & \\
\hline & 6 & $\frac{\frac{v 500}{11520}}{2}$ & $\frac{0000}{11520}$ & $\frac{2500}{256}$ & 2304 & $\frac{14001}{2304}$ & $\frac{1280}{1280}$ & $\frac{0.000}{11520}$ & $\frac{-11}{2304}$ & \\
\hline & 7 & $\frac{81227}{35840}$ & $\frac{-67681}{8960}$ & $\frac{34151}{2880}$ & $\frac{-16747}{1280}$ & $\frac{5669}{512}$ & $\frac{-76621}{11520}$ & $\frac{1699}{640}$ & $\frac{-5647}{8960}$ & $\frac{21719}{322560}$ \\
\hline \multirow{6}{*}{3} & 1 & -1 & 3 & -3 & 1 & & & & & \\
\hline & 2 & -2 & 7 & -9 & 5 & -1 & & & & \\
\hline & 3 & $\frac{-23}{8}$ & $\frac{91}{8}$ & $\frac{-71}{4}$ & $\frac{55}{4}$ & $\frac{-43}{8}$ & $\frac{7}{8}$ & & & \\
\hline & 4 & $\frac{-29}{8}$ & $\frac{127}{8}$ & -29 & $\frac{115}{4}$ & $\frac{-133}{8}$ & $\frac{43}{8}$ & $\frac{-3}{4}$ & & \\
\hline & 5 & $\frac{-8197}{1920}$ & $\frac{39139}{1920}$ & $\frac{-27219}{640}$ & $\frac{19699}{384}$ & $\frac{-15043}{384}$ & $\frac{12099}{640}$ & $\frac{-10099}{1920}$ & $\frac{1237}{1920}$ & \\
\hline & 6 & $\frac{-2317}{480}$ & $\frac{47707}{1070}$ & $\frac{-7443}{10}$ & $\frac{158471}{1020}$ & -30037 & $\frac{32091}{640}$ & -40087 & $\frac{1961}{20}$ & $\frac{-357}{61}$ \\
\hline \multirow{6}{*}{4} & & & & & & & & & & \\
\hline & 1 & 1 & -4 & 6 & -4 & 1 & & & & \\
\hline & 2 & $\frac{5}{2}$ & $\frac{-23}{2}$ & 21 & -19 & $\frac{17}{2}$ & $\frac{-3}{2}$ & & & \\
\hline & 3 & $\frac{101}{24}$ & $\frac{-87}{4}$ & $\frac{373}{8}$ & $\frac{-319}{6}$ & $\frac{273}{8}$ & $\frac{-47}{4}$ & $\frac{41}{24}$ & & \\
\hline & 4 & $\frac{287}{48}$ & $\frac{-1639}{48}$ & $\frac{1341}{16}$ & $\frac{-5527}{48}$ & $\frac{4613}{48}$ & $\frac{-783}{16}$ & $\frac{677}{48}$ & $\frac{-85}{48}$ & \\
\hline & 5 & $\frac{14861}{1920}$ & $\frac{-1447}{30}$ & $\frac{21299}{160}$ & $\frac{-25651}{120}$ & $\frac{42119}{192}$ & $\frac{-2951}{20}$ & $\frac{30437}{480}$ & $\frac{-1903}{120}$ & $\frac{1127}{640}$ \\
\hline
\end{tabular}


Acknowledgment. The comments of the referee have been most helpful in generalizing and simplifying the present algorithm.

Exxon Research and Engineering Company

Annandale, New Jersey 08801

E-mail: bfornbe@erenj.bitnet

1. M. Abramowitz \& I. A. Stegun, Handbook of Mathematical Functions, National Bureau of Standards, Appl. Math. Series, no. 55, Washington, D. C., 1964.

2. S. J. CYNAR, "Using Gaussian elimination for computation of the central difference equation coeficients," SIGNUM Newsl. (USA), v. 22, 1987, pp. 12-19.

3. B. FORNBERG, "On a Fourier method for the integration of hyperbolic equations," SIAM J. Numer. Anal., v. 12, 1975, pp. 509-528.

4. H. B. Keller \& V. PEREYRA, "Symbolic generation of finite difference formulas," Math. Comp., v. 32, 1978, pp. 955-971.

5. W. D. LAKIN, "Differentiating matrices for arbitrarily spaced grid points," Internat. J. Numer. Methods Engrg., v. 23, 1986, pp. 209-218. 